\title{
Morbilidad y Mortalidad durante el Primer Año de Vida en Pacientes con Síndrome de Down
}

\author{
NATALIA RETAMALES M. ${ }^{1}$, REGINA MORENO A. ${ }^{2}$, ANDREA GONZÁLEZ SM. ${ }^{2}$, \\ JAIME CERDA L. ${ }^{3}$, MACARENA LIZAMA C. ${ }^{1}$ \\ 1. Departamento de Pediatría, Escuela de Medicina. Pontificia Universidad Católica de Chile, Santiago, Chile. \\ 2. Servicio de Pediatría, Hospital Hernán Henríquez Aravena, Temuco, Chile. \\ 3. Departamento de Salud Pública, Escuela de Medicina. Pontificia Universidad Católica de Chile, Santiago, Chile.
}

\begin{abstract}
Down' Syndrome Patient Morbidity and Mortality during the First Year of Life

Objective: Down' Syndrome (DS) is the most common chromosomal abnormality. It carries an increased risk of concurrent congenital malformations as well as increased risk of mortality when compared to the general population. Objective: To determine the characteristics of morbidity and mortality in patients with DS during the first year of life. Patients and Methods: A prospective study of a cohort of children born with with DS was performed. All children born in 2006 in two Chilean hospitals who were given a clinical diagnosis of DS were included. Infant mortality, morbidity and nutritional diagnosis were determined and recorded every two months. Results: 33 patients were included, $78.8 \%$ were full term newborns. Average age of the mother at childbirth was $33.2 \pm 7.8$ years. Among the 33 patients, $64.5 \%$ had congenital heart disease, the most common cause being atrio-ventricular defects $(25 \%), 35 \%$ of the patients with CHD required cardiac surgery. Gastrointestinal disorders were diagnosed among $35 \%$ of these children, atresia or intestinal stenosis being the most frequent diagnosis. $25.8 \%$ of the population suffered from hypothyroidism. Follow-up showed that after two months, $54.8 \%$ of these children were malnourished, however at one year old, $22.5 \%$ were overweight. By the first year, children had shown $5.38 \pm 1.83$ episodes of Acute Respiratory Tract Infections per patient per year, and $1.22 \pm 1.14$ non neonatal hospitalizations per child/year. Half of these hospitalizations were due to lower respiratory tract infection. Two patients died during the study due to infectious disorders, resulting on a survival rate of $93.6 \%$ at 12 months. Conclusions: This information should assist physicians in improving patient follow-up. The study should also make evident the need to create national standards to supervise the care of patients with DS.

(Key words: Down syndrome, Birth defects, mortality, morbidity, infant, epidemiology, congenital heart disease).

Rev Chil Pediatr 2009; 80 (4): 323-331
\end{abstract}

Trabajo recibido el 01 de diciembre de 2008, devuelto para corregir el 08 de abril de 2009, segunda versión el 11 de mayo de 2009, aceptado para publicación el 18 de junio de 2009.

Correspondencia a:

Dra. Natalia Retamales Moreno

E-mail: nataliarmail@gmail.com 


\section{RESUMEN}

Objetivos: El Síndrome de Down (SD) corresponde a la anomalía cromosómica más frecuente, con mayor riesgo de malformaciones asociadas y mortalidad que la población general. Nuestro objetivo fue caracterizar la morbimortalidad durante el primer año de vida en pacientes con SD. Material y Método: Estudio de cohorte prospectivo en niños con SD. Se incluyeron todos los niños nacidos durante el año 2006 con diagnóstico clínico de SD en dos hospitales chilenos. Se realizó registro de morbimortalidad, hospitalizaciones y diagnóstico nutricional cada 2 meses hasta los 12 meses de vida. Resultados: Se incluyeron 33 pacientes. El 78,8\% fueron recién nacidos de término. La edad materna promedio fue de 33,2 $\pm 7,8$ años. El $64,5 \%$ presentó cardiopatía congénita, siendo más frecuentes los defectos aurículo-ventriculares (25\%). El $35 \%$ requirió cardiocirugía. Las patologías digestivas asociadas se presentaron en el 35\%. El 25,8\% presentó hipotiroidismo. A los 2 meses el 54,8\% tenía desnutrición. El 22,5\% tenía obesidad/sobrepeso a los 12 meses. Hubo un promedio de 5,38 $\pm 1,83$ infecciones respiratorias agudas (IRA) por paciente/año y 1,22 $\pm 1,14$ hospitalizaciones no neonatales por niño/año. El 50\% de las hospitalizaciones fueron por IRA baja. Dos pacientes fallecieron, ambos por causa infecciosa, con una sobrevida de 93,6\% a 12 meses. Conclusiones: El conocimiento de esta información nos permitirá mejorar el seguimiento actual de este grupo de niños, además de hacer evidente la necesidad de crear un programa nacional de evaluación y supervisión estandarizado de salud para niños con SD.

(Palabras clave: Síndrome de Down, morbilidad, mortalidad, malformación, lactante, epidemiología, cardiopatia congénita).

Rev Chil Pediatr 2009; 80 (4): 323-331

\section{Introducción}

El síndrome de Down (SD) corresponde a la anomalía cromosómica más frecuente, con una incidencia de 10-14 por 10000 recién nacidos vivos $(\mathrm{RNV})^{1,2}$. En Chile, se ha encontrado una mayor incidencia de hasta 30 por 10000 $\mathrm{RNV}^{3}$, sin encontrar una causa precisa que lo explique.

Es conocido que los pacientes con SD tienen más riesgo de presentar malformaciones asociadas $^{4}$ como cardiopatías congénitas (entre $40-50 \%)$, patología ocular (40\%), hipoacusia (40-70\%), malformaciones digestivas (20\%) y patología tiroidea (15\%), entre otras ${ }^{15,25}$, además de mayor frecuencia de enfermedades infecciosas, especialmente respiratorias, lo que se traduce en mayor morbimortalidad, y hospitalizaciones que la población general. Estudios internacionales muestran que pacientes con SD tendrían 10 veces más riesgo de hospitalización por VRS que la población general ${ }^{5-8}$.

En las últimas décadas la sobrevida ha aumentado principalmente gracias al tratamiento quirúrgico de las cardiopatías congénitas (CC), y a la implementación de programas de salud orientados al diagnóstico y tratamiento precoz de patologías asociadas ${ }^{7-9}$. Sin embargo, la sobrevida es muy diferente entre los distintos países y en comparación con la población general. Estudios en Europa y Norteamérica muestran una sobrevida de $90-93 \%$ en el primer año de vida $^{9}$, mayor a lo reportado en Sudamérica alcanzando sólo al $74 \%{ }^{10}$. Los factores de riesgo asociados a mortalidad son la presencia de malformaciones, prematurez y factores sociales como educación materna y etnia ${ }^{2,10,11}$.

En la revisión de la literatura realizada en bases de datos de Scielo y Pubmed, no encontramos trabajos prospectivos sobre morbimortalidad en niños con SD en Chile y muy escasos reportes en otros países. Nuestro objetivo es caracterizar la morbimortalidad en una cohorte prospectiva de niños con $\mathrm{SD}$, con un seguimiento a 12 meses. El conocimiento de esta información es importante tanto para optimizar los programas de salud actualmente utilizados, como para implementar nuevas estrategias de diagnóstico y tratamiento que mejoren el pronóstico en estos pacientes.

\section{Pacientes y Método}

\section{Tipo de Estudio}

Estudio de cohorte prospectivo descriptivo, 
sin intervención médica, en dos hospitales chilenos de atención terciaria de derivación neonatal: Hospital Clínico Pontificia Universidad Católica de Chile (HCPUC) y Hospital Regional Doctor Hernán Henríquez Aravena de Temuco (HHHA).

\section{Pacientes}

Se incluyeron todos los RNV nacidos entre el $1^{\circ}$ de enero y el 31 de diciembre de 2006, con diagnóstico clínico de SD confirmado por genetista según los criterios publicados por el Estudio Colaborativo Latinoamericano de Malformaciones Congénitas (ECLAMC) ${ }^{12}$. Se solicitó consentimiento informado a los padres. Fueron excluidos aquellos niños cuyos padres rechazaron participar.

\section{Registro de Información}

Al ingreso: Se registraron datos demográficos de los padres, del embarazo y parto, antecedentes familiares de SD, y en el recién nacido $(\mathrm{RN})$, patologías asociadas y hospitalización neonatal.

Seguimiento: Se registró diagnóstico nutricional, patologías asociadas al SD (CC, malformaciones digestivas, hipotiroidismo, hipoacusia, trastornos hematológicos y oculares), morbilidad, hospitalizaciones (causa y duración), cirugías y fallecimiento a los 2, 4, 6, 8, 10 y 12 meses de edad, mediante encuesta telefónica o personal efectuadas por el mismo encuestador.

\section{Definiciones}

Diagnóstico nutricional: Mediante el Índice Peso/Talla (IPT) y según curvas de crecimiento para SD publicadas por la Academia Americana de Pediatría (AAP) ${ }^{13}$, se clasificaron en eutrofia (IPT $\geq 90 \mathrm{y} \leq 110 \%$ ), sobrepeso u obesidad (IPT $>110 \%$ ) y desnutrición (IPT $<$ 90\%).

Patologías Asociadas: Corresponde a malformaciones congénitas $u$ otras enfermedades que se presentan con mayor frecuencia en niños con $\mathrm{SD}$, en comparación con la población general, según la $\mathrm{AAP}^{4}$.

Morbilidad: Todas las patologías distintas a patologías asociadas, que motivaron atención y diagnóstico por médico. Las patologías infecciosas respiratorias agudas (IRA), divididas en infección respiratoria aguda alta (IRAa) y baja (IRAb) y las patologías infecciosas no respiratorias, se clasificaron según CIE-10 ${ }^{14}$.

$C C$ : Malformaciones cardíacas estructurales, diagnosticadas por cardiólogo pediátrico mediante ecocardiografía. No se consideraron como CC significativas el ductus arterioso persistente (DAP) y foramen oval permeable descritos sólo en período neonatal, sin repercusión hemodinámica ni necesidad de tratamiento (médico y/o quirúrgico) y que en el seguimiento a 2 meses estuvieran ya resueltas. El canal aurículovetricular (canal A-V) y la comunicación interventricular (CIV) acompañada de comunicación interauricular (CIA), fueron agrupadas como defectos aurículo-ventriculares

Hipotiroidismo: Hormonas tiroideas (T3, T4 libre) bajo el rango normal o TSH sobre el rango normal para la edad, según valores de referencia respectivos. Fue considerado como congénito, cuando se diagnosticó durante el primer mes de vida.

Hipoacusia: Potenciales evocados auditivos de tronco (PEAT) o emisiones otoacústicas (EOA) alteradas uni o bilateralmente en al menos 1 de ellos.

\section{Análisis Estadístico}

La información fue registrada en tabla Excel. Se analizaron las variables mediante Stata 8.0 y Epi-info. Para la comparación de variables cuantitativas se utilizó la prueba estadística t de student. Se consideró significación estadística a un error tipo $\alpha$ de $5 \%(\mathrm{p} \leq 0,05)$. Se realizó además test de la mediana para comparación de medianas.

\section{Resultados}

Durante el período de estudio nacieron en el HCPUC un total de 7 niños con diagnóstico de SD de un total de nacimientos de 2275 (30/ 10000 RNV) y en el HHHA de Temuco 27 pacientes de un total de 7650 (35/10 $000 \mathrm{RNV})$. De los 34 pacientes que cumplieron criterios de inclusión, 1 fue excluido por rechazo de los padres a participar del estudio y en $2(6 \%)$, hubo pérdida de contacto al segundo mes de seguimiento. Estos dos últimos sólo fueron in- 
Tabla 1. Características generales de pacientes con SD

\begin{tabular}{ll}
\hline \multicolumn{2}{l}{ Características } \\
\hline $\begin{array}{l}\text { Total (n/\%) } \\
\text { Género }\end{array}$ & $33 / 100 \%$ \\
$\quad$ Masculino (n/\%) & $20 / 60,6 \%$ \\
Tipo de parto (n/\%) & $15 / 45,5 \%$ \\
$\quad$ Eutócico & $17 / 51,5 \%$ \\
$\quad$ Cesárea & $1 / 3,0 \%$ \\
$\quad$ Fórceps & \\
Edad gestacional, semanas & $37,3 \pm 2,35$ \\
(promedio \pm DS) & $7 / 21,2 \%$ \\
Edad gestacional $\leq 37$ sem (n/\%) & $2889 \pm 667$ \\
Peso, g (promedio \pm DS) & $33 / 100 \%$ \\
Apgar > 6 a los 5 minutos (n/\%) & $6 / 18,2 \%$ \\
Diagnóstico prenatal (n/\%) & $33,2 \pm 7,8$ \\
Edad materna, años (promedio \pm DS) & $7 / 21,2 \%$ \\
Antecedentes familiares de SD (n/\%) & $28 / 84,8 \%$ \\
Hospitalización neonatal (n/\%) &
\end{tabular}

cluidos para el análisis de las características generales de los pacientes al momento del ingreso al estudio, las que se muestran en la tabla 1.

El 78,8\% fueron RN de término (mayor a 37 semanas de edad gestacional) y el $87,8 \%$ tuvo un peso mayor a 2000 g. Ningún paciente requirió maniobras de reanimación avanzada al momento del nacimiento. E1 51,5\% de las madres tenían edad mayor o igual a 35 años. El $84,8 \%$ de los pacientes fueron hospitalizados en período neonatal, cuyas causas se muestran en la tabla 2. En el 80,6\% $(\mathrm{n}=25)$ se obtuvo resultado de cariograma, de ellos el 93\% ( $\mathrm{n}=$ 23) correspondió a trisomía 21 clásica, 1 a traslocación XY, der (14; 21 q10; q10), +21 y 1 a mosaico (21/25 células). Los padres del niño con traslocación fueron aconsejados por su genetista para estudio de portación de la translocación.

Las patologías asociadas a SD se muestran en la tabla 3. El estudio ecocardiográfico al nacer fue realizado en el $100 \%$ de los niños y mostró que el 58\% tenía algún tipo de CC. A excepción de 1 paciente, todos los niños con $\mathrm{CC}$ fueron hospitalizados al momento de nacer. En 2 pacientes la CC fue detectada durante el seguimiento a los 6 y 10 meses respectivamente, obteniéndose un total de 20 niños con CC
Tabla 2. Causas de hospitalización neonatal en pacientes con SD

\begin{tabular}{lc} 
Causa principal de hospitalización & n \\
\hline Total pacientes & 28 \\
Malformaciones asociadas & $18^{*}$ \\
$\quad$ Cardiopatías congénitas & 17 \\
$\quad$ Malformaciones digestivas & 4 \\
Hiperbilirrubinemia & 4 \\
Hipoglicemia & 1 \\
Síndrome dificultad respiratoria & 2 \\
Observación & 2 \\
\hline
\end{tabular}

* 3 pacientes tuvieron tanto CC como malformaciones digestivas.

Tabla 3. Patología asociada a Síndrome de Down

\begin{tabular}{lrl}
\hline Patologías asociadas $(\mathbf{n}=\mathbf{3 1})$ & $\mathbf{n}$ & $\mathbf{( \% )}$ \\
\hline Cardiopatías congénitas total & 20 & $(64,5)$ \\
Defectos aurículo-ventriculares & 5 & $(25)$ \\
CIV & 5 & $(25)$ \\
CIA & 4 & $(20)$ \\
DAP & 4 & $(20)$ \\
Tetralogía de Fallot & 1 & $(5)$ \\
Displasia válvula tricuspídea & 1 & $(5)$ \\
Patología digestivas total & 11 & $(35)$ \\
Atresia o estenosis intestinal & 5 & $(45,5)$ \\
Enfermedad de Hirshprung & 2 & $(18,2)$ \\
Ano imperforado & 1 & $(9,1)$ \\
Hipoplasia vía biliar intrahepática & 1 & $(9,1)$ \\
Enfermedad celíaca & 2 & $(18,2)$ \\
Hipotiroidismo & 8 & $(25,8)$ \\
Alteraciones oftalmológicas & 8 & $(25,8)$ \\
Hipoacusia & 10 & $(32)$ \\
Trastornos hematopoyéticos & 2 & $(6,4)$ \\
\hline
\end{tabular}

CIV: comunicación intraventricular; CIA: comunicación interauricular; DAP: ductus arterioso persistente.

(64,5\%), siendo más frecuentes los defectos aurículo-ventriculares $(25 \%)$ y CIV $(25 \%)$. Del total de niños con CC, 7 pacientes $(35 \%)$ requirieron cirugía cardíaca durante el seguimiento.

Las patologías digestivas asociadas se presentaron en 11 pacientes (35\%), siendo la atresia o estenosis intestinal, la malformación digestiva más frecuente $(\mathrm{n}=5)$. Todos ellos requirieron cirugía durante el período de RN. Dos pacientes presentaron enfermedad de Hirshprung, 1 de ellos con manifestaciones clínicas y diagnóstico desde período neonatal. En 2 pacientes fue diagnosticada enfermedad celíaca a los 8 y 12 
meses respectivamente. En todos los niños se realizó tamizaje neonatal para hipotiroidismo. En 29 pacientes se realizó al menos 1 control adicional con pruebas tiroideas durante el seguimiento. El hipotiroidismo fue detectado en un 25,8\% $(\mathrm{n}=8), 2$ de ellos congénito. A excepción de 1 paciente, a todos los niños que participaron del estudio se les realizó, al menos una evaluación auditiva, ya sea por medio de EOA o PEAT, diez pacientes cumplieron los criterios para hipoacusia durante el seguimiento. De ellos, 2 no fueron evaluados por otorrinolaringólogo durante el primer año. Los 2 pacientes con trastornos hematopoyéticos correspondieron a trastornos mielodisplásticos transitorios neonatales. Un 52\% de los pacientes fue evaluado por oftalmólogo en algún momento del seguimiento, de ellos, 8 (50\%) presentaron alguna patología ocular, entre ellas estrabismo y obstrucción del conducto lagrimal, ninguno presentó catarata congénita.

El estado nutricional de los niños durante el primer año de vida se muestra en la figura 1. El $54,8 \%$ de los niños presentaron desnutrición a los 2 meses de vida (17/31), la mayoría (14/17) correspondían a pacientes con CC.

La morbilidad ocurrida durante el seguimiento a 12 meses se resume en la tabla 4. La IRA, (código CIE-10: J00-J22), fue el diagnóstico más frecuente. Los episodios de IRA fueron estadísticamente más frecuentes en niños con $\mathrm{CC}$ (t student, $\mathrm{p}<0,001)$. Las patologías infecciosas no respiratorias correspondieron a infecciones del tracto urinario (ITU) (código CIE10: N39), síndrome diarreico agudo (SDA, código CIE-10: A01-09) y síndrome febril (código CIE-10 B99), con un promedio de 1,0 episodio por niño en 1 año. Hubo un promedio de 1,22 \pm 1,14 hospitalizaciones no neonatales por niño/ año, con una mediana de duración de 3 días (159 días), sin diferencias significativas entre el grupo con CC y $\sin \mathrm{CC}(\mathrm{p}=0,268)$. De ellas, 19 $(50 \%)$ fueron por IRAb, $11(28,9 \%)$ por cirugías (cardíacas y gastrointestinales), 4 por SDA, 2 por ITU, 1 por síndrome febril y 1 paciente por desnutrición severa.

Durante el seguimiento hubo un total de 2 pacientes fallecidos. Los antecedentes y causa de muerte se muestran en la tabla 5. En ambos, la causa última de muerte fue infecciosa $y$

Tabla 4. Morbilidad de causa respiratoria y hospitalizaciones en pacientes con SD. Comparación según presencia de cardiopatía congénita

\begin{tabular}{lcccc}
\hline $\begin{array}{l}\text { Morbilidad } \\
\text { (episodios por niño en } \mathbf{1} \text { año) }\end{array}$ & $\begin{array}{c}\text { Total } \\
(\mathbf{n}=\mathbf{3 1})\end{array}$ & $\begin{array}{l}\text { Con CC } \\
(\mathbf{n}=\mathbf{2 0})\end{array}$ & $\begin{array}{c}\text { Sin CC } \\
(\mathbf{n}=\mathbf{1 1})\end{array}$ & ** \\
\hline IRA (promedio \pm DS) & $5,38 \pm 1,83$ & $6,20 \pm 1,70$ & $3,91 \pm 0,94$ & $<0,001$ \\
IRAb (promedio \pm DS) & $1,8 \pm 0,98$ & $2,10 \pm 0,97$ & $1,27 \pm 0,79$ & $=0,022$ \\
Hospitalizaciones† (promedio \pm DS) & $0,86 \pm 0,83$ & $1,00 \pm 0,97$ & $0,55 \pm 0,52$ & $=0,163$ \\
\hline
\end{tabular}

IRA: Infección Respiratoria Aguda (alta y baja), IRAb: Infección Respiratoria Aguda Baja; *Análisis comparativo mediante t de student entre pacientes con y sin CC; † Episodios de hospitalización cuya causa fue distinta de cirugía correctora de CC o malformaciones digestivas.

Tabla 5. Características pacientes fallecidos durante el seguimiento

\begin{tabular}{|c|c|c|c|c|c|}
\hline $\mathbf{n}$ & $\begin{array}{c}\text { Edad } \\
\text { fallecimiento }\end{array}$ & $\begin{array}{l}\text { Antecedentes } \\
\text { neonatales }\end{array}$ & Patología asociada & Morbilidad & Causa de Muerte \\
\hline 1 & 1 m 21 días & $\begin{array}{l}\text { Femenino, } \\
\text { *EG } 34 \mathrm{sem} \\
(1630 \mathrm{~g})\end{array}$ & $\begin{array}{l}\text { Tetralogía de Fallot } \\
\text { con estenosis pulmo- } \\
\text { nar operada a los } 5 \\
\text { días de vida }\end{array}$ & $\begin{array}{l}\text { IRAb durante hospita- } \\
\text { lización }\end{array}$ & $\begin{array}{l}\text { Neumonía asociada a } \\
\text { atención de salud, } \\
\text { sepsis }\end{array}$ \\
\hline 2 & 8 m 5 días & $\begin{array}{l}\text { Masculino, } \\
\text { EG } 38 \mathrm{sem} \\
(3400 \mathrm{~g})\end{array}$ & $\begin{array}{l}\text { Hipoplasia vía biliar } \\
\text { intrahepática, } \\
\text { hipertensión portal }\end{array}$ & $\begin{array}{l}\text { IRAb } 3 \text { episodios hos- } \\
\text { pitalizados a los } 4,6 \\
\text { y } 8 \text { meses }\end{array}$ & $\begin{array}{l}\text { Sepsis foco pulmonar, } \\
\text { Falla multiorgánica }\end{array}$ \\
\hline
\end{tabular}

*EG: Edad gestacional; IRAb: Infección respiratoria aguda baja. 


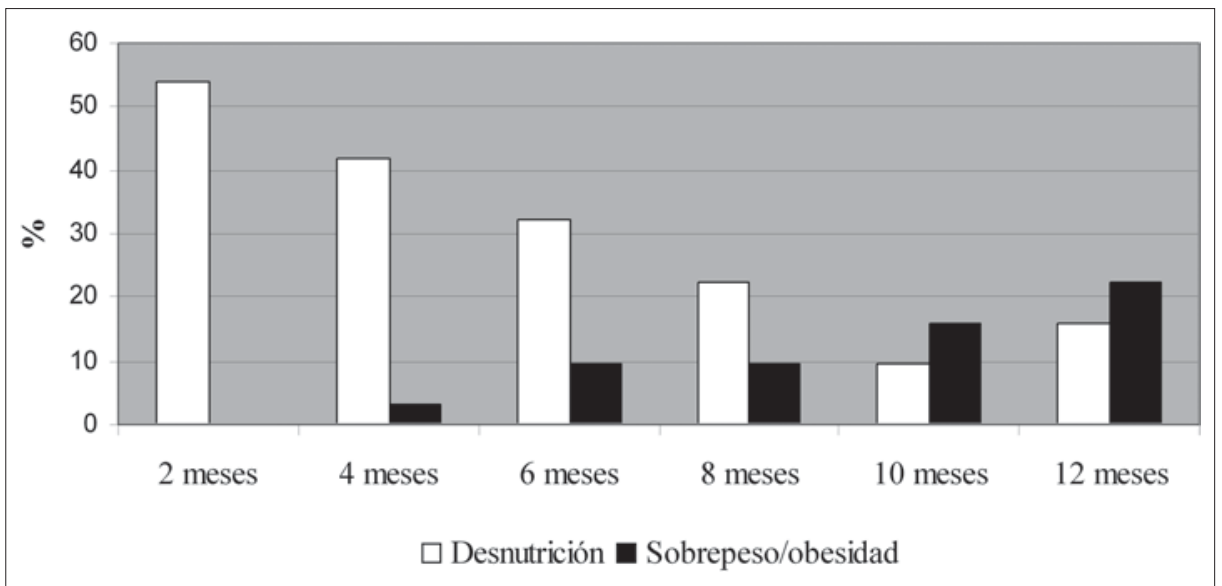

Figura 1. Desnutrición y sobrepeso/obesidad en niños con SD Según Ed. ninguno tuvo estudio de inmunodeficiencia. La sobrevida a 12 meses en esta cohorte fue de $93,6 \%$.

\section{Discusión}

Los cuidados postnatales y expectativa de vida en niños con SD han mejorado en las últimas décadas ${ }^{15}$, sin embargo, aún existen áreas en desarrollo, especialmente en prevención primaria y secundaria ${ }^{7-9}$.

En Chile, no existe registro nacional de niños con SD, sin embargo, hospitales chilenos adheridos al ECLAMC reportan una incidencia de 30 por $10000 \mathrm{RNV}^{3}$, similar a lo encontrado en nuestro estudio y ambas mayor a lo reportado en otros países ${ }^{1,2,15}$. Esto pudiera ser explicado principalmente por la mayor edad en que las mujeres chilenas se embarazan ${ }^{3,15,16}$, que en nuestro estudio es similar a lo reportado previamente en Chile ${ }^{12}$, adicionalmente pudiera existir la presencia de algún factor étnico o exposición ambiental aún no estudiada, o a la escasa utilización de screening para SD durante el embarazo mediante ecografía, gonadotropina coriónica y proteína A plasmática ${ }^{17}$, factor importante y motivo de abortos en embarazos de primer trimestre en otros países ${ }^{16}$. El hecho de que el estudio haya sido realizado en centros de referencia neonatal, no sería un factor importante, ya que al excluir a los pacientes con diagnóstico prenatal $(18,2 \%)$ la incidencia sería de 28/10 $000 \mathrm{RNV}$.
E1 80\% fueron RN de término, comparable con lo encontrado en otras series ${ }^{3,5,10,11}$, sin embargo, el $20 \%$ de prematurez es mayor a la población general chilena $(6 \%)^{18}$, de esta manera el SD pudiera condicionar mayor incidencia de resultados obstétricos adversos como rotura prematura de membranas, retraso del crecimiento intrauterino que motive un parto prematuro, entre otros ${ }^{19}$.

La hospitalización neonatal ocurrió en un alto porcentaje $(84,8 \%)$. Las recomendaciones actuales de la $\mathrm{AAP}^{4}$ no consideran la hospitalización neonatal de rutina en la evaluación del $\mathrm{RN}$ con $\mathrm{SD}^{4,20}$, sin embargo, en nuestra serie hubo 2 pacientes que se hospitalizaron para observación. A pesar de que en muchos casos este motivo de hospitalización puede ser justificado, debe tenerse en cuenta que para estos niños es fundamental facilitar y fomentar el vínculo madre-hijo.

En nuestra serie el cariograma fue realizado en el $80 \%$ de los niños, probablemente debido al alto costo del examen. Los resultados del cariograma mostraron una frecuencia de traslocación y mosaicismo similar a lo descrito en la literatu$\mathrm{ra}^{4,21}$, sin embargo, es necesario realizarlo en el $100 \%$ de los niños, como lo recomienda la $\mathrm{AAP}^{4}$, para un adecuado consejo genético.

La búsqueda de $\mathrm{CC}$ está recomendado en todos los RN dada la alta incidencia y morbimortalidad asociada. En nuestra serie destaca el alto porcentaje de CC $(64,5 \%)$ con respecto a otros estudios ${ }^{22-24}$. Dos pacientes con diagnóstico prenatal de cardiopatía fueron deriva- 
dos para nacer en el HCPUC como centro de referencia para cardiocirugía pediátrica, si se excluyeran dichos niños, la frecuencia sería del $58 \%$, ligeramente superior a lo reportado. La distribución de CC sigue el patrón habitual, con alto porcentaje de defectos atrioventriculares, CIV y CIA ${ }^{23,24}$.

Otra de las patologías que es recomendable buscar en forma activa, es el hipotiroidismo, pues su detección y tratamiento precoz mejora los resultados en el neurodesarrollo. En nuestra cohorte encontramos un alto porcentaje con patología tiroídea $(25,8 \%)$, en comparación con otros estudios ${ }^{4,25,26}$, cabe destacar que en todos se realizó al menos una evaluación de hormonas tiroídeas durante el seguimiento. La recomendación actual, durante el primer año de vida, es el estudio de hormonas tiroideas al nacer, 6 y 12 meses $^{4}$, conducta justificada, dado la importancia de su diagnóstico y tratamiento precoz.

Los trastornos auditivos y oftalmológicos fueron porcentualmente menores que lo reportado en la literatura ${ }^{4,27-30}$, probablemente debido a la dificultad en el acceso a los especialistas y al consecuente subdiagnóstico. La hipoacusia, en su mayoría de conducción, así como muchos de los trastornos oftalmológicos tienen tratamiento, por lo tanto, es deber del equipo de salud permitir que este grupo de niños tenga acceso a exámenes diagnósticos y evaluación de especialistas, de manera de corregir las patologías tratables y ofrecerles la mejor condición para expresar al máximo sus potencialidades sensoriales.

Pudimos observar un gran número de niños con desnutrición en los primeros meses de vida $(17 / 31)$, en su mayoría asociado a la presencia de CC (14/17), persistiendo $1 / 3$ de ellos con desnutrición al año. Durante los primeros meses, el sobrepeso y obesidad no fue un problema, sin embargo, a los 12 meses observamos un aumento de sobrepeso y obesidad hasta un $22,5 \%$. Este comportamiento puede ser explicado por la hipotonía, dificultad en succióndeglución, aumento del gasto energético y malformaciones asociadas (como CC), que haría de la desnutrición un tema fundamental a prevenir y tratar en los primeros meses. Por otra parte, la obesidad, que empieza a ser importan- te a finales del primer año de vida, probablemente se deba a múltiples factores como la baja actividad física, la falta de límites por parte de la familia y equipo de salud y a la utilización de curvas de crecimiento de niños sin SD, con lo que el diagnóstico nutricional y las recomendaciones de alimentación serían mal orientadas.

La morbilidad respiratoria fue las más importante con una frecuencia de de 5,38 $\pm 1,83$ / niño por año. Puesto que en nuestro estudio no fueron consideradas aquellas IRA sin diagnóstico médico, su frecuencia real podría estar subestimada. Actualmente, estudios demuestran que niños con SD tienen mayor frecuencia y severidad de infecciones respiratorias que la población general ${ }^{5,33}$, lo que puede ser explicado por alteraciones inmunológicas ${ }^{34-37}$ (no estudiadas en nuestra serie) y anatomía e hipotonía de la vía aérea, además de la mayor dificultad en la movilización de secreciones bronquia1 es ${ }^{38,39}$.

La sobrevida a 12 meses fue de $93 \%$ comparable con estudios norteamericanos y europeos, y mayor a lo reportado en Sudamérica ${ }^{10,11}$, esto probablemente debido a la mejoría en la atención de salud y a la implementación de programas nacionales para diagnóstico, tratamiento y seguimiento de $\mathrm{CC}$ y otras patologías neonatales ${ }^{40}$.

Nuestra limitante principal fue el número de pacientes reclutados, lo que pudiera sobrestimar o subestimar algunas patologías encontradas. Hubo una pérdida del seguimiento menor al 10\% del grupo seleccionado.

En nuestro país aún no existe registro nacional de nacimientos de niños con SD ni un programa nacional estandarizado de seguimiento, lo que explicaría la evaluación no uniforme ocurrida en nuestra serie y la parcial adherencia a las recomendaciones internacionales. Esto último es especialmente importante en aquellas patologías que requieren evaluación y tratamiento por especialista.

En conclusión, el conocer las causas de morbilidad, hospitalizaciones y mortalidad encontradas en esta cohorte de niños chilenos con $\mathrm{SD}$, además de hacer evidente la alta frecuencia de patologías asociadas, nos parece necesario evaluar la creación de un programa nacio- 
nal de supervisión de salud estandarizado para niños con SD, que permitan su adecuado seguimiento, pesquisa precoz de patologías asociadas y tratamiento oportuno, mejorando tanto su sobrevida como la calidad de vida de ellos y sus familias.

\section{Referencias}

1.- National Birth Defects Prevention Network: Birth defects surveillance data from selected states, 19962000. Birth Defects Res A Clin Mol Teratol 2003; 67 (9): 729-818.

2.- Centers for Disease Control and Prevention (CDC): Down Syndrome Prevalence at Birth, United States, 1983-1990. MMWR Morb Mortal Wkly Rep 1994; 43 (33): 617-22.

3.- Nazer J, Aguila A, Cifuentes L: Vigilancia epidemiológica del síndrome de Down en Chile, 1972 a 2005. Rev Méd Chile 2006; 134: 1549-57.

4.- American Academy of Pediatrics. Committee on Genetics: American Academy of Pediatrics: Health Supervision for Children With Down Syndrome. Pediatrics 2001; 107: 442-9.

5.- Bloemers B, Van Furth M, Weijerman M, et al: Down Syndrome: A Novel Risk Factor for Respiratory Syncytial Virus Bronchiolitis A Prospective BirthCohort Study. Pediatrics 2007; 120: e1076-81.

6.- Smith CS: Immune system concerns for children with Down syndrome. In: Van Dyke DC, Mattheis P, Eberly SS \& Williams J, Editors. Medical and surgical care for children with Down syndrome: A guide for parents. 1995. Woodbine House.

7.- Day S, Strauss D, Shavelle R, Reynolds R: Mortality and causes of death in persons with Down syndrome in California. Dev Med Child Neurol 2005; 47: 171-6.

8.- Leonard S, Bower C, Petterson B, Leonard H: Survival of infants born with Down's syndrome, 1980-1996. Paediatr Perinat Epidemiol 2000; 14: 163-71.

9.- Roizen NJ, Patterson D: Down's syndrome. Lancet 2003; 361: 1281-89.

10.- Castilla E, Rittler M, Dutra M, et al, and the EclamcDownsurv Group: Survival of Children With Down Syndrome in South America. Am J Med Genet 1998; 79: 108-11.

11.- Rasmussen S, Lee-Yang Wong, Correa A, Gambrell $D$, Friedman JM: Survival In Infants With Down Syndrome, Metropolitan Atlanta, 1979-1998. J Pediatr 2006; 148: 806-12.
12.- Nazer J, Cifuentes L, Ruiz G: Incidencia de 50 malformaciones congénitas en 8 Maternidades chilenas participantes del Estudio Colaborativo Latino Americano de Malformaciones Congénitas (ECLAMC). Período 1982-1988. Rev Chil Pediatr 1991; 62: 37-44.

13.- Cronk C, Crocker AC, Pueschel SM, et al: Growth Charts for Children With Down Syndrome: 1 Month to 18 Years of Age. Pediatrics 1988; 81: 102-10.

14.- Organización Mundial de la Salud: Clasificación Estadística Internacional de Enfermedades y Problemas Relacionado. Décima Revisión (CIE-10), Organización Mundial de la Salud, editores. 1995.

15.- Yang Q, Rasmussen SA, Friedman JM: Mortality associated with Down's syndrome in the USA from 1983 to 1997: a population-based study. Lancet 2002; 359: 1019-25.

16.- Collins V, Muggli E, Riley M B, Palma S B, Halliday $J$ : Is Down Syndrome a Disappearing Birth Defect? J Pediatr 2008; 152: 20-4.

17.- Malone F, Canick J, Ball R, et al: First-trimester or second-trimester screening, or both, for Down's syndrome. N Engl J Med 2005; 353 (19): 2001-11.

18.- Ministerio de Salud: Guía Clínica Prevención Del Parto Prematuro. $1^{\circ}$ Ed. Santiago: Minsal, 2005.

19.- Liu S, Lee F, Lee J, et al: Pregnancy outcomes in unselected singleton pregnant women with an increased risk of first-trimester Down's syndrome. Acta Obstet Gynecol Scand 2004; 83 (12): 1130-4.

20.- Van Cleve S, Cohen W: Part I: Clinical Practice Guidelines for Children With Down Syndrome From Birth to 12 Years. J Pediatr Health Care 2006; 20: 47-54.

21.- Hattori M, Fujiyama A, Taylor: The DNA sequence of human chromosome 21. Nature 2000; 405: 311319.

22.- García-Miñaur R, Castro V, Galdeano JM: Down's syndrome in the Basque autonomous community, 19901995: types of birth and follow-up of a cohort of 116 children during the first year of life. Registry of anomalies. An Esp Pediatr 1997; 47 (1): 61-5.

23.- Frid C, Drott P, Lundell B, Rasmussen F, Annerén G: Mortality in Down's syndrome in relation to congenital malformations. J Intellect Disabil Res 1999; 43 (Pt 3): 234-41.

24.- Freeman S, Bean L, Allen E, et al: Ethnicity, sex, and the incidence of congenital heart defects: a report from the National Down Syndrome Project. Genet Med 2008; 10 (3): 173-80.

25.- Gibson P, Newton R, Selby K, Price D, Leyland K, Addison $G$ : Longitudinal study of thyroid function in 
Down's syndrome in the first two decades. Arch Dis Child 2005; 90: 574-8.

26.- Fort P, Lifshitz F, Bellisario R: Abnormalities of thyroid function in infants with Down's syndrome. J Pediatr 1984; 104: 545-9.

27.- Roizen $N J$, Wolters C, Nicol T, Blondis TA: Hearing loss in children with Down syndrome. J Pediatr 1993; 123 (1): S9-12.

28.- Morales-Angulo C, Gallo-Terán J, Azuara N, Rama Quintela J: Otorhinolaryngo logical manifestations in patients with Down syndrome. Acta Otorrinolaringol Esp 2006; 57(6): 262-5.

29.- Da Cunha RP, Moreira JB: Ocular findings in Down's syndrome. Am J Ophthalmol 1996; 122 (2): 236-44.

30.- Fimiani F, Iovine A, Carelli R, Pansini M, Sebastio $G$, Magli $A$ : Incidence of ocular pathologies in Italian children with Down syndrome. Eur J Ophthalmol 2007; 17 (5): 817-22.

31.- Nandí-Lozano E, Espinosa LE, Viñas-Flores L, AvilaFigueroa $C$ : Infección respiratoria aguda en niños que acuden a un centro de desarrollo infantil. Salud Pública México 2002; 44 (3): 201-6.

32.- Nafstad P, Hagen JA, Oie L, Magnus P, Jaakkola JJ: Day care centers and respiratory health. Pediatrics 1999; 103 (4): 753-8.

33.- Behrendt $C$, Decker $M$, Burch D, Watson P: International variation in the management of infants hospitalized with respiratory syncytial virus. Eur J
Pediatr 1998; 157: 215-20.

34.- Murphy M, Lempert MJ, Epstein LB: Decreased level of $\mathrm{T}$ cell receptor expression by Down syndrome (trisomy 21) thymocytes. Am J Med Genet Suppl 1990; 7: 234-7.

35.- Murphy M, Hyun W, Hunte B, Levine AD, Epstein LB: A role for tumor necrosis factor-alpha and interferongamma in the regulation of interleukin-4-induced human thymocyte proliferation in vitro. Heightened sensitivity in the Down syndrome (trisomy 21) thymus. Pediatr Res 1992; 32: 269-76.

36.- Larocca LM, Lauriola L, Ranelletti FO: Morphological and immunohistochemical study of Down syndrome thymus. Am J Med Genet Suppl 1990; 7: 225-30.

37.- De Hingh YC, Van Der Vossen PW, Gemen EF: Intrinsic abnormalities of lymphocyte counts in children with down syndrome. J Pediatr 2005; 147: 744-7.

38.- Marcus C, Keens T, Bautista D, Vonpechmann W, Wardsld: Obstructive sleep-apnea in children with Down syndrome. Pediatrics 1991; 88: 132-9.

39.- Uong E, Mcdonough J, Tayag-Kier C: Magnetic resonance imaging of the upper airway in children with Down syndrome. Am J Respir Crit Care Med 2001; 163: 731-6.

40.- Ministerio de Salud: Guía Clínica Cardiopatías Congénitas Operables en menores de 15 años. $1^{\circ}$ Ed. Santiago: Minsal, 2005. 